\title{
Philippe Humblé
}

Vrije Universiteit Brussel, Belgium

\section{Arvi Sepp}

Vrije Universiteit Brussel, Universiteit Antwerpen, Boulevard de la Plaine 2, 1050 Ixelles, Belgium

\section{Dislocation linguistique et culturelle dans Yoko Tawada et Akira Mizubayashi}

\begin{abstract}
1. Introduction
Dans cet article nous nous proposons d'étudier la représentation de l'hétéroglossie dans Überseezungen (2002) de Yoko Tawada et dans Une langue venue d'ailleurs (2011) d'Akira Mizubayashi. Plus précisément, nous avons analysé les images complexes qui montrent l'interaction de forces globales et locales dans les écrits japonaisallemands de Tawada et japonais-français de Mizubayashi. En prenant pour point de départ leurs propres «lieux » transculturels, formés par des connexions et des allégeances multiples, ces deux auteurs japonais réinventent l'espace multilingue qu'ils habitent, tout en lui donnant une signification profondément personnelle, teintée par les divers courants culturels, qui sont le résultat de leur expérience.

Il est clair que pour Tawada et Mizubayashi-bien que radicalement différents pour ce qui concerne leur position littéraire et culturelle - écrire dans une langue autre que la maternelle est une
\end{abstract}


activité qui ne peut être séparée d'une quête d'identité, nouvelle ou double. L'écriture de ces deux auteurs japonais nous ouvre une fenêtre sur la relation multiculturelle complexe qui existe entre nation, langue et identité (cf. p.ex. Riley 2007). Les textes de Tawada et de Mizubayashi expriment le point de vue qu'une "authenticité » ethnique, culturelle ou linguistique n'est pas représentable et que la littérature ne peut pas être réduite à une 'unique' interprétation de l'identité.

Notre article est divisé en quatre parties. Dans la première partie, nous parlons brièvement des biographies de Tawada et de Mizubayashi en nous concentrant sur les forces thématiques sousjacentes à leurs œuvres. Dans la seconde partie, nous analysons les relations multiples entre mobilité transnationale et dislocation translinguistique dans Überseezungen de Tawada. La troisième partie traitera des formations d'identité complexes de Mizubayashi dans Une langue venue d'ailleurs, plus précisément à l'égard de la langue et de la culture françaises. Enfin, nous formulerons quelques remarques générales sur le multilinguisme, l'auto-traduction et l'idée de nation dans les œuvres des deux auteurs.

\section{Multiculturalismes Asie-Europe}

Mizubayashi, originaire du nord de Honshu au Japon, entreprend l'étude du français en 1969, à l'âge de 17 ans. Il apprend le français, dit-il, par rejet de la vacuité de la langue japonaise, telle qu'elle était maniée dans le jargon politique du mouvement étudiant dans les années soixante du siècle passé. En 1973, après avoir étudié le français, la plupart du temps en autodidacte, Mizubayashi reçoit une bourse qui lui permet de passer deux ans à Montpellier. Il retournera par après en France pour un doctorat sur Rousseau. Il y épousera aussi une française avec qui il aura une fille. Depuis 1983, Mizubayashi enseigne la littérature française à l'Université Sophia à Tokyo. Il a publié des articles académiques et, en 2011, Une langue venue d'ailleurs, un compte-rendu légèrement romancé de son " histoire d'amour » avec la langue française. En raison de son livre, Mizubayashi a reçu le Prix du Rayonnement de la langue et de la 
littérature françaises. De nombreux articles, très élogieux, ont depuis été publiés sur son ouvrage dans la presse française ${ }^{1}$. Il a publié en 2013 Mélodie : Chronique d'une passion, également en français.

Yoko Tawada, pour sa part, est une écrivain bilingue en japonais et en allemand, née en 1960 au Japon, et qui vit actuellement à Berlin. Entre ses travaux nous citons Le jeune marié était un chien (2003), Où l'Europe commence (2002), et Überseezungen (2002). Des aspects importants de son travail tournent autour de la déconstruction philosophique du langage et de l'identité. Pour son travail de création littéraire et critique, elle a reçu en 1996 -parmi de nombreux autres prix- le prestigieux prix Adalbert von Chamisso, décerné aux auteurs étrangers en reconnaissance de leur contribution à la culture allemande.

3. Mobilité transnationale et dislocation translinguistique : Überseezungen de Yoko Tawada

Dans l'écriture hétérotopique de Tawada se reflète une variété de relations possibles avec la culture allemande, ainsi que des contextes culturels, littéraires et historiques du Japon. L'importance littéraire de Überseezungen ne peut guère être appréhendée, si l'on se borne à considérer l'œuvre en relation avec un espace d'appartenance régional ou national unique. Le titre Überseezungen peut d'ailleurs être compris de deux façons, vu qu'il s'agit d'un mot composé de Übersee (outre-mer) et de Zungen (langues, en tant qu'organe). Il met l'accent sur l'intérêt de l'auteur pour les langues et les lieux étrangers. En outre, le mot Zungen énonce la dimension physique qui existe entre le langage et le corps, entre les mots et les sons.

Une seconde interprétation se base sur la proximité phonétique entre le mot Überseezungen et Übersetzungen (traductions). Dans le livre, la langue est représentée comme un espace permettant le voyage

\footnotetext{
${ }^{1}$ En voici quelques exemples (consultés le 14 avril

2014) : http://www.telerama.fr/livres/une-langue-venue-dailleurs, 64607.php; http://www.lefigaro.fr/livres/2011/04/20/0300520110420ARTFIG00650-fan-de-rousseau-fou-des-lumieres.php
} 
et la subjectivité (cf. Kraenzle 2007 : 92). Voyager et parler une langue étrangère vont de pair. L'avion, en tant qu'espace hétérotopique, devient le passage vers des lieux et des langues étrangères (cf. Ibid. : 96).

Quand je suis assise dans l'avion, j'ai à peine assez de place pour bouger mon corps. Mon dos devient raide, mes pieds et mes mollets se gonflent, mon coccyx n'est plus assis comme il devrait l'être, et ma peau se dessèche. Seule ma langue devient plus humide et plus élastique. Elle se prépare à la rencontre avec une langue étrangère ${ }^{2}$ (Tawada $2002: 115$ ).

L'adoption d'une nouvelle langue est non seulement décrite comme un voyage, mais aussi comme une métamorphose linguistique (cf. Kraenzle 2007 : 98). En effet, les voyelles et les signes divers imprègnent le corps et par conséquent transforment le sujet parlant :

Que fait-on quand on est entouré de voix étrangères ? Certains essaient, consciemment ou inconsciemment, d'adapter leurs voix au nouvel environnement. Corrige-t-on la hauteur et le volume, imite-t-on le rythme de la langue nouvelle et prête-t-on attention à l'inhalation et à l'exhalation ? Chaque consonne, chaque voyelle et peut-être chaque virgule passent à travers toutes les cellules de la chair et transforment la personne qui parle (Tawada, 1998: 8).

Überseezungen articule l'expérience multiculturelle radicale de la corporéité quand on se trouve entre deux langues et deux cultures. Tawada attribue une substance aux mots et leur prête une sensation physique. Par exemple, dans le récit «Eine leere Flasche» (Une bouteille vide) le mot 'atakushi', la forme de la première personne du singulier en japonais, qui indique un statut social privilégié, porte le parfum de Zypressenholz (bois de cyprès), l'odeur de la richesse et du confort (cf. Kraenzle, 2007 : 99). De la même manière, dans son essai «Wolkenkarte », Tawada dissèque les liens intimes qui existent entre les dialectes régionaux, la géographie et l'appartenance (cf. Ibid. : 101). Lors d'un séjour à Bâle, quelqu'un demande à l'écrivain si elle a un vélo (un Velo en suisse allemand). Ce mot déclenche une série de réflexions et d'associations qui se rapportent à nouveau à la question de l'appartenance :

\footnotetext{
${ }^{2}$ Toutes les traductions sont nôtres.
} 
J'ai été alarmée, parce que «Velo » sonne presque comme le mot japonais qui signifie 'langue'. Avez-vous une langue ? C'est une question importante. Avezvous la langue exigée pour avoir le droit d'appartenir à cet endroit ? (Tawada, $2002: 52)$.

Les langues peuvent créer un sentiment d'appartenance, mais elles peuvent aussi exclure les gens et bloquer leur entrée. D'emblée il est clair que le texte est parsemé de traductions faites par une narratrice féminine et que la traduction ne s'écarte guère d'un point fixe : la langue maternelle et la langue étrangère se modifient l'une l'autre constamment à travers les mouvements physiques de la langue, organe qui ne renferme ni os, ni noyau dur. La différence apparemment évidente entre la langue maternelle et la langue étrangère est par conséquent mise en doute par Tawada. Dans le récit «Zungentanz » (La danse des langues), un mouvement de la langue est suffisant pour entamer un procès de traduction, ce qui n'est pas sans problèmes, puisque cela comporte pertes et interférences. La langue exige constamment de nouveaux espaces. Le je se transforme et devient une langue, comme un symbole de transculturalité :

Quand je me réveille, ma langue est toujours un peu gonflée et beaucoup trop grande pour pouvoir se déplacer dans ma bouche. Elle bloque les voies respiratoires, les poumons sont mis sous pression. Je me demande durant combien de temps je dois sentir ces étouffements ? Puis ils cèdent. Ma langue me fait alors penser à une éponge usée, se retirant lentement dans l'œsophage, raide et sèche, en emportant toute ma tête (Tawada, 2002 : 9).

La narratrice s'est toujours sentie étrangère en Allemagne, parce que considérée « une étrangère qui empiète sur la langue des habitants du dehors » (Tawada 2002: 109). Elle souligne néanmoins sans cesse que la langue et la culture peuvent être adoptées et intériorisées : «J'ai avalé la langue allemande ; depuis elle s'est logée dans mon ventre. » (Ibid. : 103).

C'est précisément cet entre-les-deux (in-betweenness) que Tawada essaie d'exprimer dans ses écrits. L'authenticité des sentiments et des pensées n'est pas liée à la langue maternelle : « [C]et espace entre les deux est pour moi très important. Et [...] je tiens à écrire d'une manière qui rend cet espace entre les deux visible» (Dittberner 1994 : 197- 
198). Le traducteur est ainsi un acteur interculturel de premier plan. Dans ce contexte, l'écrivain bengali Jhumpa Lahiri, auteur de Interpreter of Maladies : Stories of Bengal, Boston and Beyond (1999) affirme également que le concept de traduction est comme un moyen de donner un sens à l'autre et à l'étranger : « La quasi-totalité de mes personnages sont des traducteurs, dans la mesure où ils doivent donner un sens à ce qui est étranger pour survivre » (Lahiri 1999: 120).

Le sens du lieu de Tawada peut être compris comme un sentiment complexe d'appartenance, conçu en reliant une multitude d'endroits différents. En tant que tel, il est un espace nuancé, malléable et dynamique. En effet, Überseezungen va au-delà de la simple fracture entre l'Allemagne et le Japon et, en mettant l'accent sur le multilinguisme, élargit la portée géographique et linguistique des quatorze textes de Überseezungen, partagés en trois sections distinctes : «Les langues eurasiennes», «Les Langues Sud-Africaines» et « Les langues de l'Amérique du Nord».

Ce faisant, Tawada complémente l'axe dominant Est-Ouest par un axe Nord-Sud (cf. Yildiz 2007 : 83). Chaque page de titre des quatorze essais montre des lambeaux de papier déchirés, noirs et blancs, qui ont la forme d'une région géographique, couverts de caractères d'alphabets étrangers (cf. Kraenzle 2007 : 92). Cette structure de l'ouvrage, ainsi que les images, reflètent les thèmes dominants de Überseezungen : la langue, la traduction, la dislocation et le moi incarné.

Dans un très beau texte de Überseezungen intitulé « Bioskoop der Nacht » (Cinéma de la nuit), Tawada met l'accent sur la dislocation de la langue, l'identité nationale, et la subjectivité. D'une manière fragmentée l'auteur oscille entre, d'une part, la description de rêves surréalistes et la tentative de répondre à la question qu'on lui pose : « dans quelle langue elle rêve ? ». La narratrice, une femme japonaise résidant en Allemagne, est frappée par le commentaire d'un parfait inconnu sur précisément cette question : "Mais on rêve dans la langue du pays dans lequel l'âme réside » (Yildiz 2007 : 83). Cette idée suppose que le rêve expose involontairement l'âme véritable d'un individu par l'intermédiaire du lien entre la langue et le territoire. 
Pourtant, le fait que la langue du rêve ne soit ni l'allemand ni le japonais, mais l'afrikaans, est très révélateur en ce qu'il annule le binaire germano-japonais. La narratrice ne veut pas que son identité en soit réduite à un seul lieu, ou à une seule langue : « J'ai beaucoup d'âmes et beaucoup de langues » (Tawada 2002 : 70). Pourtant, parce que l'Afrikaans est encore toujours associé au régime de l'apartheid, il n'offre pas d'alternative appropriée, à même de créer une sorte de troisième espace. Au lieu de cela, l'afrikaans suscite une réflexion sur le racisme et le colonialisme et la relation que le Japon maintient avec la xénophobie et ses liens avec le régime d'apartheid (cf. Yildiz 2007 : 84). Bien que la langue soit associée à l'apartheid, elle est aussi ellemême le site de l'hétérogénéité puisque, tout en étant un dérivé du néerlandais, elle porte l'influence du xhosa, de l'anglais, de l'allemand, du malais, du portugais.

Tawada déconstruit, d'un côté, l'idée romantique du monolinguisme en tant que condition nécessaire à une identité nationale et harmonieuse et, de l'autre, l'identité monolingue du sujet. Elle rejette l'hypothèse qu'il existerait une «vraie» subjectivité et que, dans l'autoréflexion, un individu ne pourrait être intimement lié qu'à une seule langue. De la même façon, Tawada critique la catégorisation de la littérature le long de frontières nationales. Les points de référence germano-japonais sont déconstruits, et non pas soulignés.

Dans ce contexte, Yoko Tawada dénonce l'intention projetée par le lecteur ou par la critique littéraire qu'elle construirait un pont multiculturel entre l'Europe et l'Extrême-Orient. Au lieu de cela, elle déconstruit et rend aliène l'expression « eine Brücke schlagen» (lancer un pont), en jouant avec les images qui lui sont associées. Ce faisant, elle renforce l'autonomie et la littérarité de ses propres textes germano-japonais :

L'expression eine Brücke schlagen, «établir un pont », me fait peur. La rive sur laquelle je me trouve soudain devient une main qui tient un bâton au-dessus de l'autre rive. De cette façon, il est contraint à un lien. Ce lien me rappelle un trait d'union : germano-français. Le premier monde et le deuxième ne peuvent être transformés en un troisième par un coup de baguette magique. Quand je me parle à moi-même, une lettre prend accidentellement la place de l'autre : a bridge or a blidge ? Dû à la mollesse de la langue, le mot sonne différemment de ce qu'il n'y 
parait. A quoi un mot ressemble-t-il de toute façon ? Il est comme un vide sous un pont. Sous le pont, la rivière se déplace dans son sommeil. Là, je rencontre des gens et je leur demande : allons-nous nous asseoir sur le rivage pour trouver une lacune dans le dictionnaire ? Faut-il ouvrir une brèche comme nous ouvrons un livre ? Ou devons-nous construire notre propre voie en nageant? (Tawada 2007a : 416).

La littérature transnationale, selon Tawada, ne peut être réduite à l'expression symbolique de collectifs (ethniques). De façon critique, la dichotomie germano-japonaise est déconstruite.

Pour en revenir au sens du titre Überseezungen, il est possible d'affirmer que la dualité langue / mobilité peut être discernée partout dans l'œuvre. Même si les frontières géographiques peuvent sembler de plus en plus insignifiantes, elles montrent comment les frontières culturelles et linguistiques demeurent intactes (cf. Kraenzle 2007 : 107).

4. Mobilité transnationale et dislocation translinguistique : Une langue venue d'ailleurs d'Akira Mizubayashi

Une langue venue d'ailleurs est un ouvrage d'un caractère entièrement différent de Überseezungen. Quand Tawada ne prétend guère être objective et aborde le sujet de l'autotraduction d'un pointde-vue littéraire, Mizubayashi nous offre le compte-rendu d'une fusion d'identités. C'est pourquoi nous nous sommes attardés plutôt à une analyse linguistique de la façon dont Mizubayashi traite, précisément, les thèmes de la «langue» et de la «traduction ». Cela, nous l'avons fait à la fois de façon explicite et de façon implicite. Explicitement, vu qu'il y a de nombreuses références directes à la «langue » en général et au «français» en particulier. Que dit Mizubayashi de ces deux entités ? D'autre part, nous avons analysé le discours de Mizubayashi de façon implicite dans le sens où l'auteur a choisi précisément «le français» comme système linguistique, comme le véhicule de son récit.

Ainsi les métaphores utilisées dans le livre de Mizubayashi pour parler du français sont assez révélatrices. Ainsi distinguons-nous la métaphore de la naissance (16 fois), du renaître, de la seconde vie, de 
respirer la langue, de vivre la langue, de s'installer dans la langue, de demeurer dans la langue, d'épouser la langue française, de maux de langue (6 fois). Ces métaphores ont trait à l'enfantement, à l'amour, à la santé et au foyer. Il ne serait guère possible de recourir à des métaphores plus vitales et, force est de le constater, plus claires. Par ailleurs, dans sa préface, Daniel Pennac dépeint Akira Mizubayashi comme étant accablé "par les 'maux de langue' que lui inflige son idiome natal.» (Mizubayashi $2011:$ 7) 'L'idiome natal' c'est le japonais, auquel Mizubayashi ne voue que peu d'affection. La langue japonaise s'oppose au français et s'obstine à empêcher une assimilation totale. Dès lors il ne faut guère s'étonner que Mizubayashi préfère utiliser le terme 'langage d'origine' au lieu de 'langue maternelle' quand il se réfère au japonais.

Pour Mizubayashi, en effet, $l e$ français n'est pas tout simplement un moyen de communication avec les français. Le français, comme langue, est l'élément de l'identité française qui peut être acquis de la façon la plus directe, qui devient une entité à part, un remède, indépendamment de sa fonction communicative. Peu de français parleraient de leur langue avec la dévotion dont fait preuve Mizubayashi :

Apprendre le français [...] est [...] le projet invraisemblable, hallucinant et gigantesque qui engage toute une existence. Le texte de Mori ${ }^{3}$ me demandait, depuis la hauteur insoupçonnée d'un discours philosophique et sur un ton austère défiant toute attitude velléitaire, si j'étais prêt à me lancer dans une telle aventure, à m'imposer une discipline de fer, à me livrer à un terrible exercice d'endurance, à m'offrir le luxe ou le risque d'une deuxième naissance, d'une seconde vie impure, hybride, sans doute plus longue, plus aléatoire, plus exposée à des ébranlements imprévisibles, plus obstinément questionneuse que la première, suffisante, autoréférentielle, peuplée de certitudes, tendanciellement repliée sur elle-même et, par cela même, parfois infatuée d'elle-même. Ma réponse fut, sans une seconde d'hésitation, oui (Ibid. : 30).

Contrairement à Tawada, qui considère qu'une langue peut être le lieu de rencontre de plusieurs cultures différentes, pour Mizubayashi

${ }^{3}$ Arimasa Mori (1911-1976), essayiste et traducteur japonais qui vécut la plus grande partie de sa vie en France. 
la langue coïncide avec une culture spécifique. Il est dès lors impossible de participer pleinement au corps français, sans une connaissance parfaite de la langue.

Lorsque nous analysons les choix lexicologiques de Mizubayashi, le style de l'auteur se révèle d'une idiomaticité impressionnante, au point de se confondre avec un français natif. En guise d'illustration, nous avons analysé un passage aléatoire quant à son degré de figement colocationnel. Pour ce faire nous avons compté le nombre de fois que des séquences «nom et adjectif » ou « substantif et verbe » avaient été utilisées dans une recherche sur Google ${ }^{4}$. En voici le résultat:

Le mois de septembre, parfois d'une accablante chaleur (G 9.320), était vite passé. Un jour il y eut un orage terrible ( $\mathrm{G} 12.900)$. Une pluie diluvienne ( $\mathrm{G}$ 45.200) tomba pendant deux heures. Le ciel zébré (G 3.970) et les grondements de tonnerre (G 128.000) signalaient le changement de saison. Le calme revenu $(\mathrm{G}$ 46.100), un majestueux arc-en-ciel (G 5.280) se dessina. C'était un spectacle grandiose (G 124.000) que je n'avais jamais vu dans les ruelles de Tokyo. (Ibid. : 143)

Nous constatons que les combinaisons utilisées par Mizubayashi sont de modérément (ciel zébré, 3.970 cas) à extrêmement usuels (grondements de tonnerre, 128.000 cas). Les adjectifs et les verbes choisis pour accompagner les substantifs sont ceux qu'un français moyen utilise quand il se met à écrire de façon « littéraire ».

D'autres exemples intéressants se trouvent dans un passage dans lequel Mizubayashi décrit les réactions des voisins de ses parents à l'occasion de l'achat d'un piano pour leur fille. L'altercation a naturellement eu lieu en japonais et la traduction est de Mizubayashi. Il est intéressant de noter que la traduction de l'auteur est du type « cibliste » ou, comme le dirait Antoine Berman la traduction offre: « un texte que l'auteur étranger n'aurait pas manqué d'écrire s'il avait écrit, par exemple, en français » (Berman 1985 : 35). Dans l'extrait qui suit, ce sont les voisins (japonais) de la famille Mizubayashi qui parlent entre eux :

- Qu'est-ce que c'est? Qu'est-ce qui se passe?

\footnotetext{
${ }^{4}$ Consulté le 10 avril 2014.
} 
- Il paraît qu'ils ont acheté un piano pour leur gosse. Vous vous rendez compte ? Un piano!

- C'est la première fois que je vois un truc pareil ! C'est énorme ! Ça a l'air vachement lourd ! Ils sont cinq!

- Ça doit coûter la peau des fesses !

- Tu parles ! Un an de salaire, il parait ! Un an, tu entends ?

- C'est de la folie ! Je ne sais pas comment ils font pour s'en sortir... La dame travaille?

- Le monsieur est professeur là-bas, au lycée !

(Ibid. : 46, les italiques sont nôtres)

Si nous préférerions utiliser la terminologie de Schleiermacher, nous pourrions dire que l'auteur laisse le lecteur «en repos » et fait l'auteur se déplacer vers lui (Schleiermacher 1813). On remarquera l'utilisation d'expressions françaises tout à fait populaires et usuelles telles que 'gosse', 'un truc pareil', 'vachement lourd', 'la peau des fesses' et 'tu parles'. Il y a eu clairement un effort de francisation du contexte japonais, et non pas de permettre au lecteur français de prendre connaissance d'une altercation typiquement japonaise.

Est-ce que Mizubayashi, comme écrivain japonais, se traduit à luimême ? Traduit-il ses propres pensées du japonais en français ? Nous pensons que non. Il n'utilise qu'une douzaine de fois les mots «traduire » et «traduction », et jamais de façon à laisser deviner une approche problématique du concept, comme ce serait le cas chez Tawada. La plupart du temps l'auteur se sert des mots « traduire» net «traduction» de façon métaphorique, comme dans «l'amour se traduit par... ». Contrairement à Tawada, pour Mizubayashi la langue coïncide avec le pays, la nation, l'identité. La langue permet d'une certaine façon d'accéder à une nouvelle identité et, aussi, de rejeter la vieille. Si dans le cas de Tawada, la langue est un objet de réflexion en soi, chez Mizubayashi cette notion n'est guère problématisée.

La langue, cependant, n'est qu'une porte d'entrée à une culture, et ne coïncide pas totalement avec celle-ci. Une personne n'entre pas dans une culture uniquement par le biais de la langue. Celle-ci n'en est 
que l'instrument, et si Yoko Tawada en est consciente, Mizubayashi aimerait qu'il en soit autrement, mais il est trop intelligent pour que des doutes ne surgissent. Même si les collocations prédominent dans le texte de Mizubayashi, celles-ci ne satisfont pas l'auteur et il est conscient que certains aspects de la langue lui seront à jamais scellés.

Mizubayashi aimerait pouvoir se convaincre qu'une nation est essentiellement constituée de langue et que, moyennant la langue, l'identité se donnerait en partage. Son texte se construit morceau par morceau, par le biais de combinaisons de mots, qui sont les mailles du filet qu'il jette sur son lecteur.

Il est important, finalement, de souligner qu'il s'agit ici du Mizubayashi qui écrit, du Mizubayashi littéraire ${ }^{5}$ et non du Mizubayashi oral. Celui-ci parvient parfaitement à être critique vis-àvis de la France et des français, mais sans que cela ne s'exprime dans son usage du français en tant que langue. Ainsi se prononce-t-il de façon très critique vis-à-vis de l'agressivité étouffée qu'il dit rencontrer parmi les français : «[...] en France, l'agressivité latente est toujours perceptible et elle peut émerger dans n'importe quelle situation, un bus bondé par exemple. » Aussi cite-t-il avec approbation Régis Debray qui dit que «[...] la société française est devenue 'tout à l'égo' » (Makhlouf 2012). N'y a-t-il pas une certaine contradiction entre la façon dont Mizubayashi écrit et la manière dont il se voit luimême en tant qu'écrivain en France ? Cela ne fait que souligner l'ambiguïté identitaire de Mizubayashi, témoin ce passage de l'interview de Makhlouf :

J'ai récemment été invité à prendre la parole aux entretiens de Royaumont et l'on m'avait donné pour thème : «Aimer la France». Or je ne suis pas sûr d'aimer la France. Je suis sûr en revanche d'aimer le français. C'est pourquoi j'ai intitulé mon intervention «Aimer la France et au-delà ». Vous savez, je suis étranger ici et là. Je suis sorti de l'univers japonais, mais je n'adhère pas pour autant à l'univers français. (Ibid.)

\footnotetext{
${ }^{5}$ Il n'est par ailleurs guère étonnant que Mizubayashi, dans sa tentative d'échapper à l'identité japonaise et d'assumer une identité française, ait choisi une forme d'expression littéraire, qui caractérise précisément le caractère national français (Pascale Casanova, 1999).
} 
Être «étranger ici et là» s'exprime dans Tawada par un distanciement de la langue d'adoption, une conviction ressentie plus que raisonnée. Dans le cas de Mizubayashi, sa façon de s'exprimer ne contredirait-il pas un peu le distanciement qu'il affiche dans ses interviews? En effet, le français ne se détache pas de la France, comme l'anglais se détache des États-Unis ou de l'Angleterre.

La problématique que les écrits de Mizubayashi illustrent est d'une nature identitaire et acquiert une dimension nouvelle dans le monde tel que nous le connaissons aujourd'hui. Alors qu'à une époque antérieure un individu comme Mizubayashi en serait réduit au silence, la mobilité lui permet aujourd'hui d'acquérir une voix et de s'exprimer. L'identité n'est plus, comme avant, imposée par la géographie.

\section{Conclusion}

Dans Imaginary Homelands (1992), Salman Rushdie examine la relation qui existe entre migration et traduction. Dans ce contexte, il explique les racines étymologiques du mot «traduction» :

Le mot « traduction » vient étymologiquement du latin et signifie «transporter d'un côté à l'autre». Après avoir été transporté à travers le monde, nous sommes des hommes traduits. [...] [Les écrivains migrants] sont capables d'écrire d'une sorte de perspective double : parce qu'eux, nous, nous sommes en même temps des initiés et des non-initiés [insiders and outsiders] de cette société » ( Rushdie $1992: 17-19)$.

Cet extrait d'Imaginary Homelands illustre un lien apparemment direct entre la mobilité et la traduction, indiquant que celle-ci est considérée comme une suite de pratiques linguistiques installées dans une situation existentielle face à la dislocation. Les notions de mobilité instaurent cette relation et la définition de Rushdie d' " hommes traduits » avec leur « vision stéréoscopique » est devenue une image emblématique de la mobilité d'aujourd'hui, du mouvement et de l'éphémère et de son implication pour l'identité individuelle et collective.

Dans le cas de Yoko Tawada, de par le fait qu'elle écrit en allemand, un univers nouveau d'associations se fait jour, créant un 
effet de dislocation et un point de référence alternatif. Le résultat est ambivalent : il nourrit un sentiment d'exotisme, d'une part, et d'autre part il montre l'importance de la langue en tant qu'instrument pour comprendre l'autre. Dans les écrits de Tawada, les protagonistes japonais recueillent leurs expériences d'hétéroglossie, les rassemblent en une mosaïque, et les dotent de valeur en établissant des liens, indépendamment du temps et de l'espace.

Tawada et Mizubayashi écrivent tous deux dans une langue étrangère, mais ils la traitent d'une façon très différente. Si dans le cas de Tawada il s'agit d'une membrane qui permet de voir le monde d'une autre façon, dans le cas de Mizubayashi c'est une peau qu'il faut s'assimiler. Les deux auteurs japonais sont des 'initiés', des insiders, mais en même temps des étrangers, des outsiders, en Allemagne comme en France. La représentation de leur identité linguistique et culturelle d'écrivains est entièrement différente. Tawada met l'accent sur l'hétérogénéité et l'élan déconstructif, et elle ne peut être réduite ni à son contexte germanique, ni à son passé japonais. Mizubayashi, par contre, cherche à s'insérer pleinement dans la tradition littéraire française, d'en faire partie, même s'il se rend compte que l'intégration complète ne se réalisera jamais.

L'aliénation et la naturalisation semblent être deux stratégies littéraires différentes pour faire face à l'expérience de l'étrangeté linguistique. En bout de ligne, cependant, la question consiste à savoir qui ou quoi a choisi la façon dont ces écrivains japonais se sont insérés dans d'autres identités : eux-mêmes, les écrivains, ou les cultures dans lesquelles ils ont voulu s'insérer?

Si les sociétés sont très différentes dans les rapports qu'ils entretiennent avec «leurs» étrangers, il est vrai aussi que, dans les deux cas qui nous occupent, il s'agit bien de deux «étrangers » qui ont choisi eux-mêmes leur lieu d'exil. Au bout du compte, Tawada a élu l'Allemagne, une société qui n'allait jamais la reconnaitre comme allemande, et elle s'en est très bien accommodée. L'Allemagne ne lui demandera jamais de prendre ses distances de son identité japonaise. En témoigne le fait que les auteurs étrangers qui écrivent en allemand ont leur propre prix : le Prix Adelbert von Chamisso. En France, par 
contre, Rousseau, Ionesco ou même Becket sont enseignés à l'école comme étant des écrivains français, et des écrivains comme Amin Maalouf, Atiq Rahimi et Fouad Bouazza ont eu droit au Prix Goncourt. C'est cette société-là que Mizubayashi a choisie et la langue est son permis de résidence. Est-ce que Une langue venue d'ailleurs nous apprend quelque chose sur la société française ? Un peu. Cela n'est pas son intention. Est-ce que Überseezungen nous apprend quelque chose sur la société allemande ? Beaucoup. Tawada reste 'in-between'. Elle observe et reste campée dans un espace à égale distance entre l'Allemagne et le Japon.

\section{Bibliographie :}

Banoun B. (2007) : «Words and Roots: The Loss of the Familiar in the Works of Yoko Tawada ", in : D. Slaymaker (dir.) Yōko Tawada. Voices from Everywhere. Plymouth, UK : Lexington Books.

Berman A. (1985) : La traduction et la lettre ou l'auberge du lointain. Les tours de Babel. Mauvezin : TER.

Casanova P. (1999) : La république mondiale des lettres. Paris : Seuil.

Clermont T. (2011) : «Fan de Rousseau, fou des Lumières », Le Figaro, en ligne, http://www.lefigaro.fr/livres/2011/04/20/03005-20110420ARTFIG00650-fan-derousseau-fou-des-lumieres.php (consulté le 14 avril 2014).

Dittberner H. (dir.) (1994) : Mit der Zeit erzählen ? fragt er'. Marcel Beyer - Heiner Egge - Gundi Feyrer - Yoko Tawada. Das zweite Buch, Göttingen : Wallstein.

Kraenzle Ch. (2007) : «Travelling without Moving : Physical and Linguistic Mobility in Yoko Tawada's Überseezungen », in : D. Slaymaker (dir.) Yōko Tawada. Voices from Everywhere. Plymouth, UK : Lexington Books.

Lahiri Jh. (1999): Interpreter of Maladies : Stories of Bengal, Boston and Beyond. New York : Mariner Books.

Landrot M. (2013) : «Une langue venue d'ailleurs», Télérama.fr, en ligne, http://www.telerama.fr/livres/une-langue-venue-d-ailleurs,64607.php) (consulté le 14 avril 2014).

Makhlouf G. (2012) : «Akira Mizubayashi, étranger à sa langue », L'Orient Littéraire, en ligne, http://www.lorientlitteraire.com/article_details.php?cid=6\&nid=3844 (consulté le 14 avril 2014).

Mizubayashi A. (2011) : Une langue venue d'ailleurs. Paris : Gallimard.

Riley P. (2007) : Language, Culture and Identity. Londres : Routledge. 
Rushdie S. (1992) : Imaginary Homelands : Essays and Criticism 1981-1991. Londres : Penguin.

Schleiermacher F. (1813): Ueber die verschiedenen Methoden des Uebersezens, en ligne, $\quad$ http://users.unimi.it/dililefi/costazza/programmi/200607/Schleiermacher.pdf (consulté le 14 avril 2014).

Senocak Z. (2000) : Which Myth Writes Me? Atlas of the Tropical Germany. Essays on Politics and Culture 1990-1998, Trad. Leslie A. Adelson. Londres : Lincoln.

Tawada Y. (2002): Überseezungen. Literarische Essays. Tübingen : Konkursbuchverlag.

Tawada Y. (2007a) : «I did not want to Build Bridges », in : D. Göktürk, D. Gramling, A. Kaes (dir.) Germany in Transit. Nation and Migration 1955-2005. Berkeley / Los Angeles / Londres : University of California Press, p. 416.

Tawada Y. (2007b) : «Tawada Yōko Does Not Exist », in : D. Slaymaker (dir.) Yōko Tawada. Voices from Everywhere. Plymouth, UK : Lexington Books, pp. 13-19.

Volle N. (2011) : « Akira MIZUBAYASHI - Une langue venue d'ailleurs », en ligne, http://enfinlivre.blog.lemonde.fr/2011/01/19/akira-mizubayashi-une-languevenue-dailleurs/ (consulté le 14 avril 2014).

Yildiz Y. (2007): «Tawada's Multilingual Moves : Toward a Transnational Imaginary », in : D. Slaymaker (dir.) Yōko Tawada. Voices from Everywhere. Plymouth, UK : Lexington Books, pp. 77-89. 